\title{
Review: pharmacological interventions are more effective than non-pharmacological for attention deficit hyperactivity disorder
}

\author{
Jadad AR, Boyle M, Cunningham C, et al. Treatment of attention-deficit/hyperactivity disorder. Evid Rep Technol Assess \\ (Summ) 1999 Nov:1-341. http://www.ahcpr.gov. \\ QUESTIONS: In patients with attention deficit hyperactivity disorder (ADHD), what are \\ the short term and long term effectiveness and safety of pharmacological and \\ non-pharmacological interventions? Are combined interventions more effective than \\ single interventions?
}

\section{Data sources}

Studies were identified by searching Medline (1966-97), CINAHL (1982-97), PsycINFO (1984-97), EMBASE/ Excerpta Medica (1984-97), and the Cochrane Library and by reviewing references of relevant studies, web sites of organisations funding research on $\mathrm{ADHD}$, and files of research team members and partner organisations.

\section{Study selection}

Studies were selected if they were randomised controlled trials (RCTs) of treatment for ADHD published in peer reviewed journals. Specific interventions considered were drug compared with drug (methylphenidate, dextroamphetamine, and pemoline), placebo compared with tricyclic antidepressant, drug compared with non-drug, combination treatments, long term treatments ( $\geqslant 12 \mathrm{wks}$ ), treatments for $\mathrm{ADHD}$ in adults, and adverse effects.

\section{Data extraction}

Data were extracted by 2 independent reviewers on 41 study elements, including methodological quality, study design attributes, funding, patient characteristics, sampling issues, diagnosis, intervention issues, and outcomes.

\section{Main results}

92 reports of 78 RCTs were included. Because substantial heterogeneity existed, a meta-analysis was not done. Among the 22 trials comparing stimulant drugs, active medications generally did not differ for effectiveness. The evidence was too limited to reliably assess the effectiveness of stimulants compared with tricyclic antidepressants. Among 6 trials comparing drug with non-drug interventions, 5 showed stimulants to be more effective than non-drug treatments. 20 trials evaluating combined interventions provided no compelling evidence to support combination treatment over stimulant or non-drug interventions alone. Among 9 trials comparing tricyclics with placebo, 6 showed a benefit of desipramine, whereas the 3 trials evaluating imipramine showed inconsistent results. 14 drug and non-drug trials had long term treatment periods (12 to $60 \mathrm{wks}$ ). A trend toward general improvement over time was shown regardless of treatment. Results of 12 trials evaluating the treatment of $\mathrm{ADHD}$ in adults were inconclusive. The study with the highest methodological score showed more benefit with methylphenidate than with placebo. 29 trials included assessment of such adverse effects as sleep disorders, headaches, tics, decreased appetite, abdominal pain, irritability, nausea, and fatigue. No evidence was available for more severe, long term adverse effects, such as risk for addiction with stimulants, liver toxicity with pemoline, or cardiac arrhythmias with antidepressants.

\section{Conclusions}

In patients with $\mathrm{ADHD}$, published studies of treatment regimens provide limited information of effectiveness because of small sample sizes, methodological flaws, and heterogeneity across outcomes. Pharmacological interventions are consistently more effective than nonpharmacological interventions. Combined interventions do not show greater benefit than single interventions.

\section{COMMENTARY}

This careful look at the evidence on the treatment of ADHD by Jadad et al confirms current clinical practice in North America. The methods were sound, and potential bias that was introduced by using only published studies was counterbalanced by the frequent communication between the research team and ADHD experts who were likely to know of important unpublished work. One RCT, the Multimodal Treatment Study of Children with Attention-Deficit/ Hyperactivity Disorder (MTA), is key. It was published after this AHRQ report was released, but the authors extracted data from the manuscript accepted for publication. It is the only RCT that is both large and well designed. ${ }^{2} 579$ children were followed for 14 months in 4 treatment conditions. Overall results are in keeping with this review: psychostimulants are superior to behavioural treatment or community care. Combined medication and behavioural treatment do not offer substantial improvement over medication alone in controlling ADHD symptoms.

2 issues from the MTA study need to be highlighted to make the results more useful to clinicians and to amplify the conclusions of the review by Jadad $e t$ al. Firstly, monthly monitoring and adjustment of psychostimulants in the study group was important: two thirds of children in community health care received psychostimulants but no formal monitoring protocol, and they did not do as well. Secondly, behavioural treatment added benefit over psychostimulants alone for some non-ADHD symptom areas. These included oppositional or aggressive symptoms, internalising symptoms, social skills, parent-child relations, and the presence of comorbid anxiety.

The MTA study findings are based on clinical practice in North America. In Europe, psychostimulants are usually reserved for severe cases and are used 10 to 30 times less often than in the US. ${ }^{3}$

What accounts for such a great difference? Is this reflective of differences in cultural attitudes toward medicating children, drug budgets, or diagnostic thresholds, to mention only 3 possibilities? How do we best define "outcome," and whose definitions are better? The rewards of good work are more questions. 